\title{
Pseudo Jahn-Teller mechanism for symmetry-breaking phase transitions in vortex molecules
}

\author{
L. F. Chibotaru, ${ }^{1}$ G. Teniers, ${ }^{2}$ A. Ceulemans,${ }^{1}$ and V. V. Moshchalkov ${ }^{2}$ \\ ${ }^{1}$ Afdeling Kwantumchemie, Katholieke Universiteit Leuven, Celestijnenlaan 200F, B-3001 Leuven, Belgium \\ ${ }^{2}$ Nanoscale Superconductivity and Magnetism Group, Laboratorium voor Vaste-Stoffysica en Magnetisme, Katholieke Universiteit Leuven, \\ Celestijnenlaan 200D, B-3001 Leuven, Belgium
}

(Received 26 April 2004; published 13 September 2004)

\begin{abstract}
The phase transitions from a symmetric superconducting order parameter to a different or broken symmetry phase are investigated for thin mesoscopic superconductors well below the nucleation temperature. By using an effective two-state model the mechanism of these transitions has been revealed and four distinct types of transitions have been found. The symmetry-breaking phase transition has the same structure as the pseudo Jahn-Teller instability of high symmetry nuclear configurations in molecules. This analogy provides an interesting connection between real and vortex molecules. The existence of broken-symmetry phases is predicted to be strongly dependent on the size of the samples.
\end{abstract}

DOI: 10.1103/PhysRevB.70.094505

PACS number(s): 74.78.Na, 74.20.De, 74.25.Ha

Vortex matter in nanostructered superconductors shows a number of new properties in comparison with the bulk reference materials. ${ }^{1-5}$ In particular, the superconducting condensate turns out to be strongly influenced by the geometry of the sample. Similarly, Bose-Einstein condensates also show a strong dependence on the geometry of their confinement. ${ }^{6}$ The nucleation of superconductivity in applied magnetic field, $T_{c}(H)$, is described by the lowest level $\epsilon_{1}$ of the linearized Ginzburg-Landau (LGL) equation. This is always nondegenerate for finite size samples, ${ }^{7}$ therefore it is fully consistent with the symmetry of the sample in applied field. ${ }^{8}$ Examples of such symmetrical $(\mathrm{S})$ solutions are giant vortex states in disks. ${ }^{3,4}$ This is opposite to the case of bulk type-II superconductors without boundaries, where $\epsilon_{1}(H)$ is an infinitely degenerate Landau level while the nucleating order parameter is a combination of its degenerate components, ${ }^{9}$ always of broken symmetry (BS) type. Besides the symmetry, the discreteness of the spectrum of the LGL equation in mesoscopic superconductors implies the stability of the shape of the nucleated order parameter in a range of temperatures close to the $T_{c}(H)$ line. Such stability of a symmetric order parameter has been found for mesoscopic cylinders, ${ }^{10-12}$ squares and triangles. ${ }^{8,13-16}$ Remarkably, a similar phenomenon is encountered in molecular physics where it is known as the pseudo-Jahn-Teller effect. ${ }^{17}$

In this paper we investigate the mechanisms of phase transitions from a nucleated order parameter of a mesoscopic superconductor to another symmetry or broken symmetry phase when temperature is lowered. We find that in the case of BS phase transitions most often only one single LGL solution of different symmetry effectively admixes to the nucleated phase. In this case the description of the phase transition is equivalent to the description of vibronic instability in a simple (two-level) pseudo Jahn-Teller problem. Such analogy is specific to mesoscopic superconductors, which have discrete LGL spectrum, and gives a "molecular" view on the mechanism of BS phase transitions in mesoscopic samples. We have also investigated the existence of different phases as a function of the samples size and found that the region on the phase diagram corresponding to the nucleated order parameter of S-solution increases with reducing the size. The critical sizes, corresponding to the disappearence of BS phase transitions (when the nucleated $\mathrm{S}$-phases persist down to $T=0$ ) are predicted to be in the range of micrometers for conventional superconductors, i.e., within the reach of current experimental techniques. This opens new possibilities for the experimental verification of different transitions predicted here on the basis of the similarities with the pseudo Jahn-Teller mechanism.

Consider a superconducting polygon of size $a\left(a^{2}\right.$ is the surface of the sample) and thickness $d$ in a perpendicular uniform magnetic field $H$. For small $(a \sim \xi$, the coherence length) and thin $(d \ll \xi)$ samples one can neglect the variation of the order parameter across thickness ${ }^{10}$ and the distortion of the magnetic field induced by screening and vortex currents. The order parameter $\Psi$ is found from the twodimensional GL functional:

$$
\Delta F=\int\left[\alpha|\Psi|^{2}+\frac{1}{2} \beta|\Psi|^{4}+\frac{1}{2 m^{*}}\left|\left(-i \hbar \nabla-\frac{2 e}{c} \mathbf{A}\right) \Psi\right|^{2}\right] d S
$$

where $\mathbf{A}$ is vector potential of the applied field and $\alpha$ $=\alpha^{\prime}{ }_{T}\left(T-T_{c}\right) \equiv-\hbar^{2} / 2 m^{*} \xi(T)^{2}$. $\Psi$ obeys in addition the appropriate boundary condition. ${ }^{18}$ Minimizing (1) without the term $\sim|\Psi|^{4}$ results in the linear eigenvalue equation:

$$
\frac{1}{2 m^{*}}\left(-i \hbar \nabla-\frac{2 e}{c} \mathbf{A}\right)^{2} \phi_{i}=\epsilon_{i} \phi_{i}
$$

Its lowest solution $\phi_{1}$ describes the nucleation phase boundary via the equation $\epsilon_{1}=-\alpha$. The eigenvalues $\epsilon_{i}$, measured in units of $\hbar^{2} / 2 m * a^{2}\left(\epsilon_{i}^{\prime}\right)$, depend only on the applied magnetic flux $\Phi=H a^{2}$, presented in units of the superconducting flux quantum $\Phi_{0}$. The eigenstates of (2), normalized to unity within the surface of the sample, are used further as the basis set for the order parameter, 


$$
\Psi=\sum_{i=1}^{N} c_{i} \phi_{i}
$$

where $N$ is the dimension of the basis set. Substitution of (3) into (1) yields $\Delta F$ as a function of the expansion coefficients:

$$
\begin{gathered}
\Delta F=\sum_{i} \alpha_{i}\left|c_{i}\right|^{2}+\frac{\beta}{2 a^{2}} \sum_{i j k l} A_{i j}^{k l} c_{i}^{*} c_{j}^{*} c_{k} c_{l}, \\
\alpha_{i} \equiv \alpha+\epsilon_{i},
\end{gathered}
$$

where the parameters

$$
A_{i j}^{k l}=a^{2} \int \phi_{i}^{*} \phi_{j}^{*} \phi_{k} \phi_{l} d S
$$

depend only on the geometry but not on the size of the sample. $A_{i i}^{i i}$ is precisely the Abrikosov parameter $\beta_{A}$ (Ref. 18) for the state $\phi_{i}$, which is a measure of its "flatness."

The actual parameters defining the relative free energy in Eq. (4) can be found as follows. If we use new coefficients $c_{i} \rightarrow a \sqrt{-\alpha_{1} / \beta} c_{i}$ and measure the free energy in units of $a^{2} \alpha_{1}^{2} / \beta$ ( $\alpha_{1}$ corresponds to the lowest LGL eigenvalue $\left.\epsilon_{1}\right)$ then the right-hand side of Eq. (4) will depend (besides $A_{i j}^{k l}$ ) only on the ratios $\alpha_{i} / \alpha_{1}=\left(a^{2} / \xi^{2}+\epsilon_{i}^{\prime}\right) /\left(a^{2} / \xi^{2}+\epsilon_{1}^{\prime}\right)$. Hence the GL functional for a given sample (measured in units of $\left.a^{2} \alpha_{1}^{2} / \beta\right)$ and the emerging phase diagram are only dependent on $(a / \xi(T))^{2}$ and $\Phi / \Phi_{0}$.

The spectrum of eigenvalues in (2) is strongly influenced by the symmetry of the problem. If the sample has a rotational symmetry axis $C_{n}$, the eigenstates are characterized by $n$ different one-dimensional irreps which transform as $\sim \exp (\operatorname{im} \varphi), m=0,1, \ldots, n-1$ under rotations around this axis. As a result the Landau levels are split in groups of $n$ levels belonging to different irreps, because only these can intersect each other (lower panel in Fig. 1). In addition, the $C_{n}$ symmetry imposes the selection rules on integrals in Eq. (5), $m_{k}+m_{l}-m_{i}-m_{j}=0$, similar to the case of cylindrical symmetry. ${ }^{11}$

Because the fourth order terms in (4) are overall positive, it is generally expected that only the states $\phi_{i}$ with $\alpha_{i}<0$ will effectively contribute to the order parameter. In the close vicinity to the nucleation phase boundary only $\alpha_{1}$ is negative, therefore $\Psi \approx c_{1} \phi_{1}$, with $c_{1}=a \sqrt{-\alpha_{1} / \beta A_{11}^{11}} \sim \sqrt{(a / \xi)^{2}-\epsilon_{1}^{\prime}}$ and the free energy $\Delta F_{1}=-\left(\alpha_{1}^{2} / 2 \beta A_{11}^{11}\right) a^{2}$. The only allowed admixtures in this phase are from the excited LGL states of the same irrep., $\phi_{1^{\prime}}$, described by the coefficients $c_{1^{\prime}}$ :

$$
\frac{c_{1^{\prime}}}{c_{1}} \approx-\frac{A_{11^{\prime}}^{11} c_{1}^{2}}{\alpha_{1^{\prime}} / \beta+\left[2 A_{11^{\prime}}^{11^{\prime}}+\left|A_{11^{\prime}}^{11}\right| \cos \left(\chi_{1^{\prime} 1^{\prime}}^{11}-2 \chi_{11^{\prime}}^{11}\right)\right] c_{1}^{2}},
$$

where $\chi_{i j}^{k l}=\arg A_{i j}^{k l}$. In the temperature region where $\alpha_{1^{\prime}}>0$ the coefficients $c_{1}$, in (6) show smooth behavior, with continuous derivatives with respect to temperature. The symmetry-preserving phase transitions can therefore occur only when some of $\alpha_{1}$, become negative. However in symmetric samples the $\phi_{i}$ states which are close to $\epsilon_{1}$ are always of different symmetry (lower panel in Fig. 1) so that many of the corresponding parameters $\alpha_{i}$ will become negative before

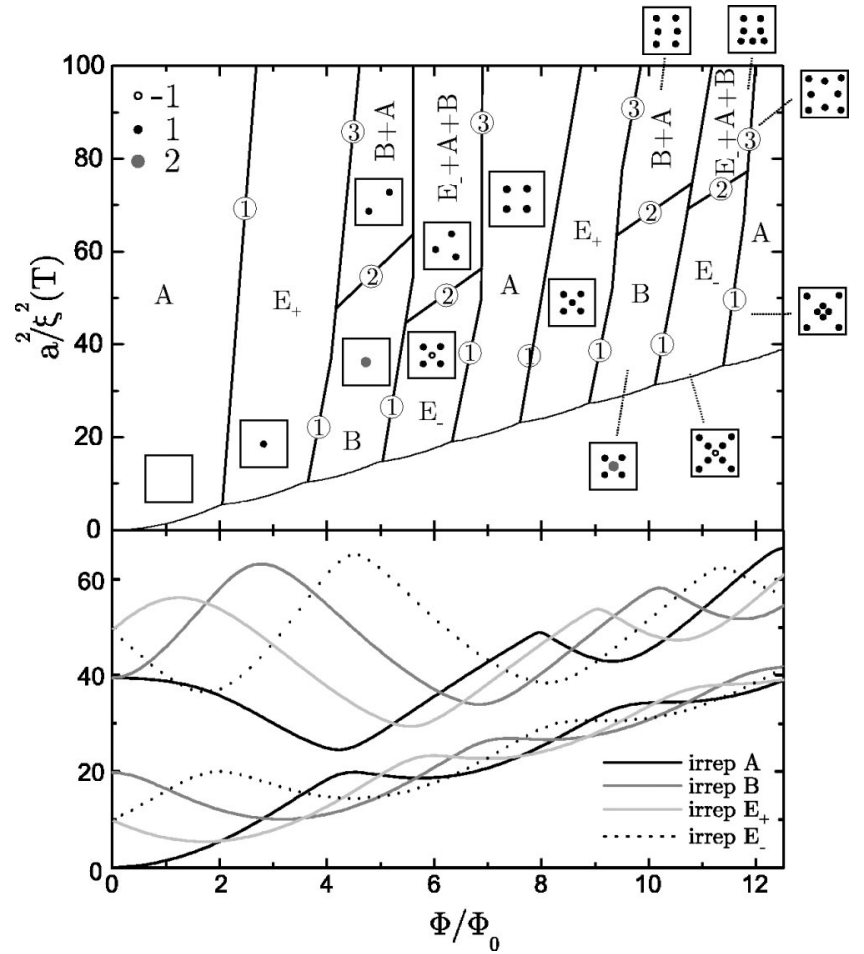

FIG. 1. (Color online) Lower panel: LGL solutions $\epsilon_{i}^{\prime}$ for a square with superconductor-vacuum boundary condition, characterized by irreps $A(m=0), B(m=2), E_{+}(m=1)$, and $E_{-}(m=-1)$. Upper panel: the corresponding phase diagram obtained by Monte Carlo calculations. For each phase, the vortex structure is shown schematically and the involved irreps are indicated. The number at each boundary line denotes the type of transition (Table I). In the color online version: the colors stand for the winding number of the central vortex: 0 (yellow), 1 (green), 2 (blue), and -1 (red).

$\alpha_{1}$. We can conclude that the nucleated order parameter will undergo a phase transition modifying its symmetry when temperature is lowered.

Even if there are many LGL states with $\alpha_{i}<0$ at a given temperature, only a few of them actually contribute to the order parameter. This is due to the fact that while the terms $\sim A_{i i}^{i i}$ and $\sim A_{i i}^{j j}$ give net contributions to "repulsion," the other terms, which could become negative, partially cancel out when $N$ is increased. One can check indeed that already for $N>3$ there are less available phases of complex $c_{i}$ coefficients than $A_{i j}^{k l}$ terms to be optimized. The mutual reduction of these terms increases with the number of mixed LGL states which means that at a certain value of $N$ further admixure will become unfavorable. It is expected therefore that only a few different irreps will effectively admix at the transition point.

Next we adopt a general description of the phase transitions from a nucleated order parameter, which is achieved by the following consideration. Given the small number of different irreps among the states $\phi_{i}$ which admix at the transition point, we can always divide the corresponding $\left\{c_{i}\right\}$ in two groups so as to bring the functional (4) to the following basic form: 


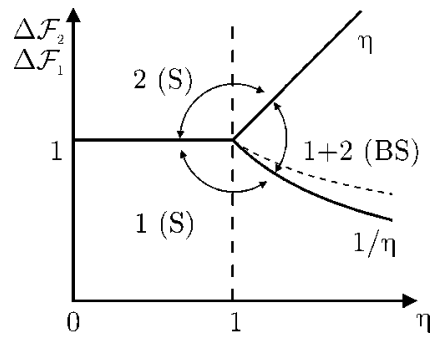

FIG. 2. Diagram of thermodynamically stable phases (solid lines) for the effective two-state model (7) as function of the parameters from Eqs. (8) and (9). 1, 2 are pure phases and 1+2 is the mixed one.

$$
\Delta F=\widetilde{\alpha}_{1} c_{1}^{\prime 2}+\widetilde{\alpha}_{2} c_{2}^{\prime 2}+\frac{\beta}{2 a^{2}}\left(A_{11} c_{1}^{\prime 4}+A_{22} c_{2}^{\prime 4}+2 A_{12} c_{1}^{\prime 2} c_{2}^{\prime 2}\right)
$$

where $c_{1}^{\prime}$ and $c_{2}^{\prime}$ are the norms of the coefficients in the first and the second group, respectively, while the parameters $\widetilde{\alpha}_{1}$, $\widetilde{\alpha}_{2}(<0)$ and $A_{11}, A_{22}, A_{12}(>0)$ are functions of associated angular variables to be specified below. The two groups contain different irreps, in numbers $n_{1}$ and $n_{2}$, correspondingly ( $\phi_{1}$ belongs to the first group). Minimization with respect to $c^{\prime}{ }_{1}$ and $c^{\prime}{ }_{2}$ for fixed values of the five parameters in (7) results in three (meta)stable phases:

$$
\begin{gathered}
\Delta F_{1}=-\frac{\tilde{\alpha}_{1}^{2}}{2 \beta A_{11}} a^{2}, \quad \Delta F_{2}=-\frac{\tilde{\alpha}_{2}^{2}}{2 \beta A_{22}} a^{2}, \\
\frac{\Delta F_{12}}{\Delta F_{1}}=\frac{\left(\sqrt{\eta \Delta F_{2} / \Delta F_{1}}-1\right)^{2}}{\eta-1}+1,
\end{gathered}
$$

where

$$
\eta=\frac{A_{11} A_{22}}{A_{12}^{2}} .
$$

The first two are pure phases, with $c_{2}^{\prime}=0$ and $c_{1}^{\prime}=0$, respectively, while $\Delta F_{12}$ is the mixed one $\left(c_{1}^{\prime}, c_{2}^{\prime} \neq 0\right)$. Figure 2 shows the diagram of the thermodynamically stable phases. The vertical line $\eta=1$ divides the diagram in two regions. On the left-hand side we have a switch between pure phases (first-order transition). On the right-hand side the two phase boundary lines correspond to the second order transitions. At temperatures close to the lower phase boundary, $T=T_{\mathrm{BS}}-\Delta T, \quad \xi=\xi_{\mathrm{BS}}-\Delta \xi$, the $\mathrm{BS}$ phase will grow as $c_{2}^{\prime} \sim \sqrt{\Delta T}, \sqrt{\Delta \xi}$.

Now the free energy expressions (8) are minimized with respect to the remaining variables from $\widetilde{\alpha}_{i}$ and $A_{i j}$ resulting in the lowest energy phase for a given temperature. Since we are looking for phase transitions from the nucleated S-order parameter, the pure phase 1 in Fig. 2 always corresponds to $\phi_{1}$ with possible small admixures of the same symmetry, Eq. (6). Depending on the symmetries of other LGL states which admix through the transition we can have several types of phase transitions which are investigated below. As in the case of $\phi_{1}$, the contributions from states of other symmetries are mainly represented by one LGL state. Therefore to simplify further analysis we will consider that only one state per irrep. contributes.

In the case of a single admixed state $\left(\phi_{2}\right)$ one should substitute $c_{1}=c_{1}^{\prime}$ and $c_{2}=c_{2}^{\prime}$ in Eq. (7) and $\tilde{\alpha}_{i}=\alpha_{i}, A_{i i}=A_{i i}^{i i}$, and $A_{12}=2 A_{12}^{12}-\left|A_{11}^{22}\right|$ into (8) and (9). When the interaction between these states, $A_{12}$, is larger than $\sqrt{A_{11}^{11} A_{22}^{22}}$, the order parameter corresponds either to $\phi_{1}$ or $\phi_{2}$ (left side of the diagram in Fig. 2). The transition from $\phi_{1}$ to $\phi_{2}$ takes place when

$$
\frac{\alpha_{2}}{\alpha_{1}}>\sqrt{\frac{A_{22}^{22}}{A_{11}^{11}}}
$$

The left-hand side of this equation increases with lowering the temperature, being always $<1$. Therefore the transition between symmetric states can only occur if $A_{22}^{22}<A_{11}^{11}$. When the interaction is weaker, $\eta>1$, the transition from $\phi_{1}$ to a $\mathrm{BS}$ order parameter (right-hand side of the phase diagram) can arise under the condition

$$
\frac{\alpha_{2}}{\alpha_{1}}>\frac{A_{12}}{A_{11}^{11}}
$$

When two states of different symmetry mix with $\phi_{1}$, two situations can occur.

(1) If the involved irreps. obey the inequalities $m_{1}+m_{3}$ $-2 m_{2} \neq 0, \pm n, \quad m_{1}+m_{2}-2 m_{3} \neq 0, \pm n, \quad$ then $\quad c_{1}=c_{1}^{\prime}, \quad c_{2}$ $=c_{2}^{\prime} \cos \varphi, c_{3}=c_{2}^{\prime} \sin \varphi$, and

$$
\begin{gathered}
\tilde{\alpha}_{1}=\alpha_{1}, \\
\tilde{\alpha}_{2}=\alpha_{2} \cos ^{2} \varphi+\alpha_{3} \sin ^{2} \varphi, \\
A_{11}=A_{11}^{11}, \\
A_{22}=A_{22}^{22} \cos ^{4} \varphi+A_{33}^{33} \sin ^{4} \varphi+\left(A_{23}^{23}-\left|A_{22}^{33}\right| / 2\right) \sin ^{2} 2 \varphi, \\
A_{12}=2 A_{12}^{12} \cos ^{2} \varphi+2 A_{13}^{13} \sin ^{2} \varphi-\left|A_{11}^{23}\right| \sin 2 \varphi .
\end{gathered}
$$

Substituting (12) into Eqs. (8) and minimizing with respect to $\varphi$ we obtain again three thermodynamically stable phases of Fig. 2 for corresponding equilibrium values of $\varphi$. The difference is that now the left-hand side of the diagram describes the switch between the symmetric $\left(\phi_{1}\right)$ and the broken-symmetry $\left(\phi_{2}+\phi_{3}\right)$ phases, while the BS phase in the right-hand side of the diagram corresponds to $\phi_{1}+\phi_{2}+\phi_{3}$.

(2) If the first or the second relation for irreps. becomes equality then $c_{1}=c_{1}^{\prime} \cos \varphi, c_{2}=c_{2}^{\prime}, \quad c_{3}=c_{1}^{\prime} \sin \varphi$, or $c_{1}$ $=c_{1}^{\prime} \cos \varphi, c_{2}=c_{1}^{\prime} \sin \varphi, c_{3}=c_{2}^{\prime}$, respectively. Therefore for $\eta<1$ we can only have a symmetry-changing transition from $\phi_{1}$ to $\phi_{2}$ (or $\phi_{3}$ ). However for $\eta>1$, at $\varphi$ corresponding to thermodynamically stable BS phase $\phi_{1}+\phi_{2}+\phi_{3}$, the lowest boundary line in Fig. 2 separates this phase from the metastable one $\phi_{1}+\phi_{3}$ (or $\phi_{1}+\phi_{2}$ ). Therefore the phase transition from $\phi_{1}$ will take place along the line which lies somewhere higher (dashed line in Fig. 2), i.e., it is of the first order. This type of transition is associated with a small jump of $c_{1}$, hence it is close to second order. 
TABLE I. Possible transitions from a symmetric vortex phase.

\begin{tabular}{cccccc}
\hline \hline Type $^{\mathrm{a}}$ & $\eta$ & $n_{1}$ & $n_{2}$ & $n_{f}^{\mathrm{b}}$ & Order (symmetry) \\
\hline 1 & $<1$ & 1 & 1 & 1 & I (S) \\
2 & $>1$ & 1 & $\geqslant 1$ & $n_{2}+1$ & II (BS) \\
3 & $<1$ & 1 & $>1$ & $n_{2}$ & I (BS) \\
4 & $>1$ & $>1$ & $\geqslant 1$ & $n_{2}+n_{1}$ & I (BS) \\
\hline \hline
\end{tabular}

${ }^{\text {aNumbers used in Fig. } 1 .}$

${ }^{b}$ Number of irreps. in the final state.

Considering higher numbers of mixing irreps will not result in qualitatively new phase transitions which are thus of four types (Table I).

The diagram of the lowest transitions from symmetric phases in a thin square was evaluated within the above approach, which compares well with an accurate Monte Carlo calculation $^{19}$ shown in the upper panel of Fig. 1. The critical values of $\xi$ calculated by the two approaches differ by only several percent. One finds indeed that only a few states effectively admix to the order parameter. The described region in the phase diagram becomes relatively large with decreasing $a$. For small enough samples some phase boundary lines pass above $(a / \xi(0))^{2}$ and the nucleated symmetric phases remain thermodynamically stable down to $T=0$. Thus the transition from the phase with an antivortex in the center $\left(\Phi / \Phi_{0}=5.5 \div 6.5\right)$ to a BS phase with the same vorticity but without antivortex is supressed for $a<(7 \div 8) \xi(0)(\approx 1 \mu \mathrm{m}$ for $\mathrm{Al}$ ).

It follows from Fig. 1 that the phase boundary lines separating the areas with different vorticity have positive slopes and correspond to transitions of type 1 (Table I) in the lower part of the diagram. ${ }^{20}$ The reason is the increase of Abrikosov parameters $\beta_{A}$ in the lowest group of LGL states (Fig. 1) when passing through the corresponding avoided crossings towards increased fields. Indeed, it was shown ${ }^{21}$ that the lowest Landau level of each irrep. maps into cylindrical states with rotational numbers $L$ to the left and $L+n$ to the right of the avoided crossing, respectively. Therefore for two lowest LGL states the Abrikosov parameter is smaller for the ground state to the left and for the excited one to the right of their intersection, so that the condition (10) can only be obeyed in the latter case.

On the other hand the obtained transitions to brokensymmetry phases are always of the second order and go mostly via a two-state mixing scenario (in the phases $E_{-}$ $+A+B$ in Fig. 1 the admixure of $B$ states is relatively small). The direct analogy for this in molecular physics is the pseudo Jahn-Teller (PJT) instability of symmetric geometry of a molecule with respect to a low symmetry nuclear distortion $(q)$. Usually such an instability results from a strong interaction of the ground electronic state $\left(\phi_{1}\right)$ with an excited state $\left(\phi_{2}\right)$, induced by $q$, which is described by the Hamiltonian: ${ }^{17}$

$$
H_{\mathrm{PJT}}=\frac{1}{2} K q^{2}+\left(\begin{array}{cc}
-\Delta & V q \\
V q & \Delta
\end{array}\right),
$$

where $2 \Delta$ is the energy gap between the ground and the excited states in the symmetric nuclear configuration, $V$ is
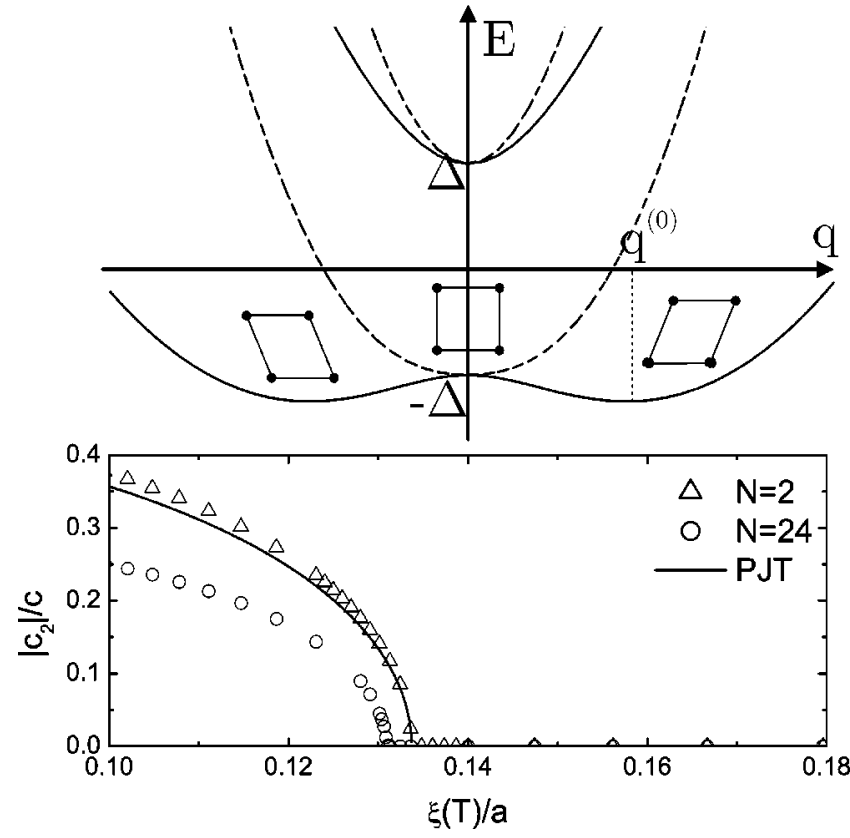

FIG. 3. Upper panel: adiabatic potential for two nondegenerate electronic terms of a square molecule in the case of weak (dashed lines) and strong (solid lines) pseudo Jahn-Teller effect. Lower panel: temperature dependence of the normalized coefficient of admixure of the excited $(A)$ state close to the $B \rightarrow B+A$ transition at $\Phi=4.5 \Phi_{0}$ (Fig. 1) evaluated by Monte Carlo calculations and the pseudo Jahn-Teller effect using the correspondence relations (16). The numbers in the inset denote the dimension of the basis set in the Monte Carlo calculations.

the vibronic constant, and $K$ is the force constant. The instability occurs when $V^{2} / K>\Delta$ and it results in an equilibrium distortion $q^{(0)}$ (Fig. 3) and a broken-symmetry electronic ground state.

The PJT instability can be described by considering a functional depending on electronic variables only. ${ }^{22}$ To obtain such a functional, we average $H_{\mathrm{PJT}}$ over $\Psi=c_{1} \phi_{1}$ $+c_{2} \phi_{2}$, find the equilibrium value of $q$ as function of $c_{1}$ and $c_{2}$ and substitute it back into the average:

$$
\left\langle\Psi\left|H_{\mathrm{PJT}}\right| \Psi\right\rangle^{0}=-\frac{2 V^{2}}{K} c_{1}^{2} c_{2}^{2}+\Delta\left(-c_{1}^{2}+c_{2}^{2}\right) .
$$

Next we introduce polar coordinates, $c_{1}=c \cos \varphi, c_{2}$ $=c \sin \varphi$ for the PJT functional (14) and $c_{1}=A_{11}^{-1 / 4} c \cos \varphi$, $c_{2}=A_{22}^{-1 / 4} c \sin \varphi$ for the functional (7), where $\varphi$ plays now the role of the order parameter for the BS state. The $\varphi$-dependent part of both functionals has now the following common form:

$$
\Delta E(\varphi)=-A c^{4} \sin ^{2} 2 \varphi-B c^{2} \cos ^{2} \varphi
$$

where $A=V^{2} / 2 K, B=\Delta$ for the PJT problem and $A$ $=\left(\beta / 4 a^{2}\right)\left(1-\eta^{-1 / 2}\right), B=\left(\alpha_{2} A_{22}^{-1 / 2}-\alpha_{1} A_{11}^{-1 / 2}\right) / 2$ for the case of superconductor. The main difference between them is that $c=1$ in the former and $c \neq 1$ in the second case. Therefore the correspondence between PJT and GL parameters is the following: 


$$
\begin{gathered}
V^{2} / K \rightarrow \frac{\left|\alpha_{1}\right|}{2 \sqrt{A_{11}}}\left(1-\frac{1}{\sqrt{\eta}}\right), \\
2 \Delta \rightarrow \frac{\alpha_{2}}{\sqrt{A_{22}}}-\frac{\alpha_{1}}{\sqrt{A_{11}}} .
\end{gathered}
$$

Lower panel in Fig. 3 shows how the broken symmetry phase appears for vortex molecules. We can see that the evolution of the order parameter with temperature is reproduced by the pseudo Jahn-Teller effect. In the case $N=2$, only the ground $B$ and the first excited $A$ LGL states are taken into account. The accurate calculation involving $N=24$ LGL states shifts the transition point obtained in the $N=2$ calculation only by $\approx 2 \%$. This shift mainly arises due to the renormalization of the effective parameters in Eq. (7) when more LGL states are taken into account. As a result of this renormalization the nucleated phase becomes optimized so that the transition takes place at a lower temperature compared to the two-state approximation.

As the analysis shows the specific structural similarity between the pseudo Jahn-Teller and GL symmetry breaking mechanisms is due to the presence of a quartic dependence on the expansion coefficients. A different mechanism of symmetry breaking was described by Berger for the case of the Schrödinger equation for a cylinder. ${ }^{23}$ In this case breaking of axial symmetry was obtained through the induced magnetic field and the quartic term in the GL potential was not considered.

In conclusion, we have investigated the mechanisms of the phase transitions in symmetric mesoscopic superconductors from a nucleated order parameter. Compared to bulk type II superconductors where only the Abrikosov vortex lattice occurs, the mesoscopic samples show a rich variety of novel vortex phases. By using an effective two-state model the nature of the transition to these phases has been revealed and four distinct types of transitions have been found. The symmetry-breaking phase transition has the same structure as the pseudo Jahn-Teller instability of high symmetry nuclear configurations in molecules. This analogy provides an interesting connection between real and vortex molecules.

The existence of novel phases can be experimentally verified by using various local probe techniques such as Hall probe microscopy, STM and AFM. The phase diagram is found to be strongly dependent on the samples size. In particular, the region on the phase diagram corresponding to the nucleated (symmetric) order parameter enlarges with reducing the size of the sample. The critical size corresponding to the complete disappearence of the BS phase (the nucleated $\mathrm{S}$-phase persists until $T=0$ ) is predicted to be of the order of micrometers for conventional superconductors, such as $\mathrm{Al}$, $\mathrm{Pb}$. These predictions can be checked experimentally on different mesoscopic superconducting systems.

Financial support from the Belgian Government (Ministerie van het Wetenschapsbeleid) and the Belgian National Science Foundation (FWO), and the KU Leuven Research Fund GOA/2003/03 and GOA/2004/02 Programme is acknowledged.
${ }^{1}$ V.V. Moshchalkov, L. Gielen, C. Strunk, R. Jonckheere, X. Qiu, C. Van Haesendonck, and Y. Bruynseraede, Nature (London) 373, 319 (1995).

${ }^{2}$ A.K. Geim, I.V. Grigorieva, S.V. Dubonos, J.G.S. Lok, J.C. Maan, A.E. Filippov, and F.M. Peeters, Nature (London) 390, 259 (1997).

${ }^{3}$ D. Saint-James, Phys. Lett. 15, 13 (1965).

${ }^{4}$ V.V. Moshchalkov, X.G. Qiu, and V. Bruyndoncx, Phys. Rev. B 55, 11793 (1997).

${ }^{5}$ A.K. Geim, S.V. Dubonos, J.G.S. Lok, M. Henini, and J.C. Maan, Nature (London) 396, 144 (1998).

${ }^{6}$ A.L. Fetter, J. Low Temp. Phys. 129, 263 (2002).

${ }^{7}$ Except for the points of accidental degeneracy where levels of different symmetry intersect.

${ }^{8}$ L.F. Chibotaru, A. Ceulemans, G. Teniers, and V.V. Moshchalkov, Physica C 369, 149 (2002).

${ }^{9}$ A.A. Abrikosov, Sov. Phys. JETP 5, 1174 (1957).

${ }^{10}$ V.A. Schweigert, F.M. Peeters, and P. Singha Deo, Phys. Rev. Lett. 81, 2783 (1998); V.A. Schweigert and F.M. Peeters, Phys. Rev. B 57, 13817 (1998).

${ }^{11}$ J.J. Palacios, Physica B 256-258, 610 (1998); Phys. Rev. Lett. 84, 1796 (2000).

${ }^{12}$ G.F. Zharkov, Phys. Rev. B 63, 224513 (2001).
${ }^{13}$ J. Bonca and V.V. Kabanov, Phys. Rev. B 65, 012509 (2002); T. Mertelj and V.V. Kabanov, ibid. 67, 134527 (2003).

${ }^{14}$ B.J. Baelus and F.M. Peeters, Phys. Rev. B 65, 104515 (2002).

${ }^{15}$ A.S. Mel'nikov, I.M. Nefedov, D.A. Ryzhov, I.A. Schereshevskii, V.M. Vinokur, and P.P. Vysheslavtsev, Phys. Rev. B 65, 140503 (2002).

${ }^{16}$ V.R. Misko, V.M. Fomin, J.T. Devreese, and V.V. Moshchalkov, Phys. Rev. Lett. 90, 147003 (2003).

${ }^{17}$ I.B. Bersuker and V.Z. Polinger, Vibronic Interactions in Molecules and Crystals (Springer, Berlin, 1989).

${ }^{18}$ A.A. Abrikosov, Fundamentals of the Theory of Metals (NorthHolland, Amsterdam, 1988).

${ }^{19}$ G. Teniers, V.V. Moshchalkov, L.F. Chibotaru, and A. Ceulemans (unpublished).

${ }^{20}$ Interestingly, phase boundary lines with negative slopes have been found for a thin superconducting film with a circular hole: A. Bezryadin, A. Buzdin, and B. Pannetier, Phys. Rev. B 51, 3718 (1995).

${ }^{21}$ L.F. Chibotaru, A. Ceulemans, M. Lorenzini, and V.V. Moshchalkov, Europhys. Lett. 63, 159 (2003); 63, 476(E) (2003).

${ }^{22}$ A. Ceulemans, J. Chem. Phys. 87, 5374 (1987).

${ }^{23}$ J. Berger, Phys. Rev. B 63, 172507 (2001). 